\title{
BVRI Photometry of 27 Kuiper Belt Objects with ESO/Very Large Telescope ${ }^{\star, \star \star}$
}

\author{
A. C. Delsanti ${ }^{1,2}$, H. Boehnhardt ${ }^{1}$, L. Barrera ${ }^{3}$, K. J. Meech ${ }^{4}$, T. Sekiguchi ${ }^{1,5}$, and O. R. Hainaut ${ }^{1}$ \\ 1 European Southern Observatory, Casilla 19001, Santiago, Chile \\ 2 Observatoire de Paris-Meudon, 5 place Jules Janssen, 92195 Meudon Cedex, France \\ 3 Universidad Catolica del Norte, Antofagasta, Chile \\ 4 Institute for Astronomy, 2680 Woodlawn Drive, 96822 Honolulu, Hawaii \\ 5 National Astronomical Observatory of Japan, Osawa, Mitaka, Tokyo 181-8588, Japan
}

Received 12 September 2001 / Accepted 10 October 2001

\begin{abstract}
We present visible ( $B V R I)$ photometric measurements of 27 Trans-Neptunian objects, obtained with the FORS2 instrument on the ESO/8 m Very Large Telescope (Unit 2) from runs during September and November 2000. The objects display a broad and continuous range of colors from neutral-bluish to very red. Most of the objects also have a linear reflectivity spectrum over the $V R I$ range. There is no evidence of a bimodal color distribution as has been previously reported by other groups. Several objects (1994 TB, $1995 \mathrm{SM}_{55}$, $1998 \mathrm{UR}_{43}, 1999 \mathrm{CF}_{119}$ and $2000 \mathrm{OK}_{67}$ ) show evidence of changing color, and should be investigated further. The object $1995 \mathrm{SM}_{55}$ deserves a more complete study since its neutral-blue colors suggest it could be a good candidate for hosting cometary activity.
\end{abstract}

Key words. Kuiper Belt - techniques: photometry - minor planets, asteroids

\section{Introduction}

The region of the Edgeworth-Kuiper Belt, beyond the orbit of Neptune, is the origin of icy bodies that are among the most pristine solar system objects observable from Earth: Trans-Neptunian Objects (TNOs), which are still located in that region, and Centaurs, which are now found in the Saturn-Neptune region. The TNOs are subdivided into three dynamical classes: classical objects (also called Cubewanos, due to the first object discovered $1992 \mathrm{QB}_{1}$ ), Resonant objects (also named Plutinos, in reference to the most famous of them, Pluto) and Scattered Disk objects (Minor Planet Center 2001b). Centaurs are now located closer to the Sun because of recent (i.e. within the last few million years; Levison 2001) gravitational scattering by Neptune and the other giant planets, or ejection by collisions among TNOs. As of 11 September 2001, 431 TNOs (Minor Planet Center

Send offprint requests to: A. Delsanti,

e-mail: adelsant@eso.org

* Based on observations performed with the Very Large Telescope facility of the European Southern Observatory at Cerro Paranal, Chile.

** Tables 2 and 3 are also available at CDS via anonymous ftp to cdsarc.u-strasbg.fr $(130.76 .128 .5)$ or via http://cdsweb.u-strasbg.fr/cgi-bin/qcat?J/A+A/380/347 2001c) and 79 Centaurs and Scattered Disk Objects (Minor Planet Center 2001a) have been discovered mainly through dedicated search programs.

Studies of the physical nature of TNOs and Centaurs are based mostly on broadband colors and low-dispersion spectroscopy in the visible and near-IR. With the exception of the few largest objects, the only size estimates available are those computed from coarse single or two color photometry (necessarily assuming a value of the albedo, in general 4\%, Keller 1990) obtained by the search programs. More accurate multi-color photometry, suitable for a first taxonomic classification of the objects, is published for roughly $15 \%$ of the known TNOs and about $40 \%$ of the Centaurs: in absolute numbers, to date, 64 TNOs and 11 Centaurs (for a summary of the available results, discussions and references see Barucci et al. 1999 and Boehnhardt et al. 2001 or Hainaut \& Delsanti 2001 for a more complete list). TNOs and Centaurs exhibit a broad range of colors, which may be indicative of the different processing of their surfaces. The existence of a bimodal color distribution (Tegler \& Romanishin 1998) appears to be controversial (Barucci et al. 2000; Davies et al. 2000; Boehnhardt et al. 2001; Hainaut \& Delsanti 2001). Distance and inclination dependent color diversities (Tegler \& Romanishin 2000; 
Trujillo et al. 2001) have not been firmly established yet, since they are based on small number statistics. Trends for a more complex surface color taxonomy of TNOs are indicated in a statistical color mode analysis (Barucci et al. 2001). From the observations published for Centaurs (in total 11 objects) at best the likely color range can be estimated (Boehnhardt et al. 2001) and comparisons between the Centaurs and other populations (comets and TNOs) are not yet conclusive (Hainaut \& Delsanti 2001), although Boehnhardt et al. (2001) suggest that the spectral gradient population of TNOs and Centaurs may be different.

Spectra of TNOs and Centaurs are even more sparse in the literature; the spectra of TNOs in the visible ( $<10$ objects) are featureless and their spectral slope is in agreement with the reddening predicted from the photometric colors of the objects (see Boehnhardt et al. 2001 and references therein, and Sect. 5.4 for a more detailed description of the reddening and how it is obtained). Most of the TNOs measured spectroscopically in the near-IR ( $\sim 10$ objects) display featureless spectra even though their reddening is very different from each other. However, absorption bands of water and hydrocarbons were found in one TNO in the near-IR (1996 $\mathrm{TO}_{66}$; Brown et al. 1997, 1999; Noll et al. 2000), but may also exist to a lesser extent in other TNO spectra (Brown 2001). Five objects have been spectroscopically observed in both visible and nearIR (Barucci et al. 1999) and revealed mostly reflectance spectra. In the $H$ and $K$ band, water ice and tholin-like absorptions were also reported, such as for $1996 \mathrm{TO}_{66}$ (Brown et al. 1999; Noll et al. 2000), or Pholus (Davies et al. 1993; Cruikshank et al. 1998). The identification of a very weak and wide silicate absorption from $I$ to $J$ band is tentative only (Cruikshank et al. 1998). In the case of Chiron, CN gas emission has also been detected at $388 \mathrm{~nm}$ (Bus et al. 1991) in addition to the water ice absorption bands in the IR reported by Luu et al. (2000).

At present, it is still difficult to obtain conclusive results on the color taxonomy and the relationship between surface colors and spectral signatures of TNOs and Centaurs, since the number of objects measured is low and does not allow clear and statistically significant results.

In this paper we present new, accurate broadband color photometry of 24 TNOs and 3 Centaurs, as a significant contribution to the published data set; we discuss the individual observations of these objects and compare them to previously published data, when available.

\section{Observations}

The CCD photometry of TNOs and Centaurs was performed at the Very Large Telescope (VLT) facility of the European Southern Observatory in Chile. During two runs in Sep. and Nov. 2000, visible images of the objects through broadband filters were taken at Unit Telescope 2 (UT2) with the FOcal Reducer instrument (FORS2). For the BVI bands, Bessell filters were used.
For the $R$ band, a special interference filter was installed, to provide about the same central wavelength and equivalent bandwidth as the Bessel $R$ filter, but with a slightly smaller transmission range with sharp wings, in order to avoid strong atmospheric emission lines. For details on the telescope and instrumentation, refer to ESO (2001a, b). A summary of the observing conditions during both runs is given in Table 1 .

The objects were selected for their observability, and their contribution to new and/or improved broadband colors in the visible and near-IR wavelength ranges. In addition, objects were selected such that there would be no bright ( $<16$ mag) field stars near the expected target and with minimal field galaxy contamination. JHK photometry of a subset of the $B V R I$ targets was executed simultaneously at the $V L T$ telescope UT1 with the near-IR focal reducer instrument ISAAC. The results of the nearIR observations will be communicated and discussed in a separate paper (Boehnhardt et al., in preparation).

The $B V R I$ exposures of a single object were typically executed within 20 to $60 \mathrm{~min}$. For the short sequences, a set of $R B V I R$ exposures were taken; for longer ones (more than $30 \mathrm{~min}$ ) the $R$ filter was repeated about every $30 \mathrm{~min}$ in between the whole filter series, in order to monitor possible rotational brightness variations of the object due to rotation. Sidereal tracking (which introduced a trailing $<0.6^{\prime \prime}$ i.e. of the order of the seeing during the observations), auto-guiding and full active optics corrections were used.

For the image calibration, standard star fields from Landolt (1992), sky flats and bias exposures were taken.

\section{Data reduction}

The data were processed using MIDAS (Munich Image \& Data Analysis System, ESO 2000). The images were biassubtracted and divided by a flat-field. The flat-field frame was either a combination of twilight flats or a "hyperflat". For the first run (i.e. Sep. 2000), the final flat-field used was simply a median frame of a series of normalized, bias-subtracted twilight flat-field images. For the second run, this could be further improved by a hyper-flat-field frame created for each filter. The long science exposures were normalized to unit sky background and median averaged, to produce a night sky flat-field. We then combined the different orders of the wavelet transformation of $i$ ) the twilight median flat-field frame and ii) the night sky flatfield frame. This process allowed us to keep the best information on high and low frequencies of the twilight and night flat-field frames. This method is described in detail in Hainaut et al. (1998). At this stage, we did not correct images for bad columns or cosmic rays; instead, we prefer to only correct the blemishes present in the immediate neighborhood of the object when necessary. 
Table 1. Summary of observing conditions for the 2 runs at ESO/Very Large Telescope. The objects observed during these runs are listed in Table 2.

\begin{tabular}{lll}
\hline UT date & $\begin{array}{l}\text { Telescope + Instr. } \\
\text { Filters }\end{array}$ & $\begin{array}{l}\text { Sky/Seeing } \\
\text { Observers }\end{array}$ \\
\hline \hline $4-5$ Sep. 2000 & VLT UT2 + FORS2 & Photometric $/ 0.8-1.8^{\prime \prime}$ \\
& $B V I($ Bessell $), R($ special $)$ & Barrera, Boehnhardt, Sekiguchi \\
& & \\
$28-30$ Nov. 2000 & VLT UT2 + FORS2 & Photometric $/ 0.4-1.2^{\prime \prime}$ \\
& $B V I$ (Bessell $), R($ special $)$ & Barrera, Boehnhardt, Meech \\
\hline
\end{tabular}

\section{Photometry}

\subsection{Classical photometry}

The photometric calibration was done using the MIDAS TMAG package (Hainaut 2001). The Landolt standard stars were measured using a large (i.e. $15^{\prime \prime}$ diameter) aperture. The zero point of the telescope, the extinction coefficients and the color correction terms were computed for each run by least square fit for the standard stars.

Using the MIDAS magnitude/circle function, the object was measured with an aperture diameter set to 4 times the stellar full width half maximum $(F W H M)$ for each individual frame. This $F W H M$ was evaluated on each image from several nearby, non-saturated stars. The sky background was measured in a 10 pixel (i.e. $2^{\prime \prime}$ ) wide annulus centered on the object, at $\sim 6 F W H M$ from its center. The results obtained with this method, for all the objects, are summarized in Table 2.

For some of the faintest objects, the contribution of the sky background noise became important when using the default $4 F W H M$ diameter aperture. For these cases, we overcome this problem by using a $3 F W H M$ diameter aperture. The resulting magnitudes were not adjusted for an aperture correction and as we use these magnitudes for color study, the resulting colors are not affected either. The quantitative effect of this change is discussed in the next section.

\subsection{Aperture correction}

We performed an analysis of the aperture photometry in order to estimate the validity of the results from the previous section. To do so, we estimated the total magnitude of a high $\mathrm{S} / \mathrm{N}$ star on each frame from the asymptotic value of its photometric growth profile. This value was compared in each case with that of the $4 F W H M$ aperture photometry. The correction appears to be constant from filter to filter and is estimated to be $0.03 \mathrm{mag}$ for a 4 FWHM aperture. We therefore consider that the colors presented in this paper, resulting from the magnitudes listed in Table 2, are not affected by the aperture effect. However, it must be noted that the absolute magnitudes are too faint by $\sim 3 \%$.

\section{Results}

\subsection{Color indexes}

The resulting color indexes computed from the classical photometry measurements are shown in Table 3. For objects that have been measured at different epochs, we present one value per epoch in order to monitor any intrinsic brightness variation between the two runs. For objects which have repeated $R$ measurements during the same night of observation, the colors are obtained from the average $R$.

\subsection{Absolute magnitude}

The absolute magnitude $M(1,1)$ has been computed for each epoch. It is defined as:

$M(1,1)=R-5 \log (r \Delta)$,

where $R$ is the $R$ magnitude, $r$ and $\Delta$ are respectively the helio- and geocentric distances $[\mathrm{AU}]$ at the time of the observations. We do not take into account the phase correction $-\beta \alpha$, where $\beta$ is the phase coefficient (cf. Meech \& Jewitt 1987 and Delahodde et al. 2001 for values of phase coefficient; a typical value is $\left.\beta=0.04 \mathrm{mag} /{ }^{\circ}\right)$, and $\alpha$ is the phase angle. Indeed, $M(1,1)$ is used in this paper only for a rough estimation of the radius of the object, whose error is dominated by the albedo uncertainty.

As for color indexes, for objects with several epochs available, we present one value of the $M(1,1)$ obtained per epoch. In this way, we can improve our interpretation of any brightening or change between two epochs.

\subsection{Radius}

Assuming a canonical surface albedo of $p=0.04$ (corresponding to that of cometary nuclei, Keller 1990), we can estimate the radius $R_{N}[\mathrm{~km}]$ of each object. This value of the albedo is in agreement with thermal IR measurements obtained for TNOs by Thomas et al. (2000). $R_{N}[\mathrm{~km}]$ is computed with the following formula (Russell 1916):

$p R_{N}^{2}=2.235 \times 10^{16} \times 10^{0.4\left(M_{\odot}-M(1,1)\right)}$, 
Table 2. Magnitudes obtained for Run 1 and 2 using a fixed aperture diameter. $r$ [AU] is the heliocentric distance at the epoch of the observations.

\begin{tabular}{|c|c|c|c|c|c|c|c|}
\hline Object & UT date & $r[\mathrm{AU}]$ & $B$ & $V$ & $R$ & $I$ & Notes \\
\hline $1993 \mathrm{SB}$ & 2000 Nov. 30 & 30.46 & $\begin{array}{c}24.11 \pm 0.09 \\
-\end{array}$ & $\begin{array}{c}23.35 \pm 0.05 \\
-\end{array}$ & $\begin{array}{l}22.88 \pm 0.08 \\
22.84 \pm 0.07\end{array}$ & $\begin{array}{c}22.34 \pm 0.09 \\
-\end{array}$ & \\
\hline 1994 ТВ & 2000 Sep. 05 & 29.60 & $\begin{array}{c}23.84 \pm 0.06 \\
-\end{array}$ & $\begin{array}{c}22.74 \pm 0.04 \\
-\end{array}$ & $\begin{array}{l}22.04 \pm 0.06 \\
21.89 \pm 0.05\end{array}$ & $\begin{array}{c}21.24 \pm 0.06 \\
-\end{array}$ & \\
\hline & 2000 Nov. 30 & 29.54 & $\begin{array}{c}23.89 \pm 0.11 \\
-\end{array}$ & $\begin{array}{c}22.85 \pm 0.05 \\
-\end{array}$ & $\begin{array}{l}22.28 \pm 0.08 \\
22.24 \pm 0.08\end{array}$ & $\begin{array}{c}21.56 \pm 0.11 \\
-\end{array}$ & \\
\hline $1995 \mathrm{SM}_{55}$ & 2000 Sep. 05 & 39.41 & $\begin{array}{c}21.34 \pm 0.01 \\
-\end{array}$ & $\begin{array}{c}20.69 \pm 0.01 \\
-\end{array}$ & $\begin{array}{l}20.30 \pm 0.02 \\
20.31 \pm 0.02\end{array}$ & $\begin{array}{c}20.01 \pm 0.03 \\
-\end{array}$ & \\
\hline & 2000 Nov. 29 & 39.39 & $21.20 \pm 0.06$ & $20.56 \pm 0.03$ & $20.23 \pm 0.05$ & $19.88 \pm 0.07$ & \\
\hline $1995 \mathrm{TL}_{8}$ & 2000 Nov. 30 & 42.42 & $\begin{array}{c}22.57 \pm 0.07 \\
-\end{array}$ & $\begin{array}{c}21.54 \pm 0.03 \\
-\end{array}$ & $\begin{array}{l}20.85 \pm 0.06 \\
20.82 \pm 0.06\end{array}$ & $\begin{array}{c}20.19 \pm 0.06 \\
-\end{array}$ & \\
\hline $1996 \mathrm{RQ}_{20}$ & 2000 Sep. 05 & 39.48 & $\begin{array}{c}24.38 \pm 0.13 \\
-\end{array}$ & $\begin{array}{c}23.43 \pm 0.07 \\
-\end{array}$ & $\begin{array}{l}22.93 \pm 0.09 \\
22.70 \pm 0.07\end{array}$ & $\begin{array}{c}22.27 \pm 0.10 \\
-\end{array}$ & 2,4 \\
\hline $1997 \mathrm{QH}_{4}$ & 2000 Nov. 30 & 41.20 & $24.96 \pm 0.16$ & $23.86 \pm 0.06$ & $23.34 \pm 0.09$ & $22.68 \pm 0.13$ & \\
\hline $1997 \mathrm{QJ}_{4}$ & 2000 Sep. 05 & 34.31 & - & $\begin{array}{c}23.04 \pm 0.11 \\
-\end{array}$ & $\begin{array}{l}22.62 \pm 0.08 \\
22.55 \pm 0.08\end{array}$ & $\begin{array}{c}22.19 \pm 0.08 \\
-\end{array}$ & 5 \\
\hline $1998 \mathrm{BU}_{48}$ & 2000 Nov. 29 & 27.50 & $\begin{array}{c}23.16 \pm 0.07 \\
-\end{array}$ & $\begin{array}{c}22.05 \pm 0.03 \\
-\end{array}$ & $\begin{array}{l}21.39 \pm 0.06 \\
21.42 \pm 0.06\end{array}$ & $\begin{array}{c}20.84 \pm 0.07 \\
-\end{array}$ & 1,3 \\
\hline $1998 \mathrm{SG}_{35}$ & 2000 Sep. 05 & 9.85 & $21.72 \pm 0.02$ & $21.03 \pm 0.01$ & $20.57 \pm 0.02$ & $20.04 \pm 0.03$ & \\
\hline $1998 \mathrm{SM}_{165}$ & 2000 Nov. 29 & 34.74 & $\begin{array}{c}22.86 \pm 0.06 \\
-\end{array}$ & $\begin{array}{c}21.93 \pm 0.03 \\
-\end{array}$ & $\begin{array}{l}21.29 \pm 0.05 \\
21.28 \pm 0.05\end{array}$ & $\begin{array}{c}20.64 \pm 0.06 \\
-\end{array}$ & \\
\hline $1998 \mathrm{SN}_{165}$ & 2000 Sep. 05 & 38.16 & $22.25 \pm 0.10$ & $21.69 \pm 0.06$ & $21.20 \pm 0.06$ & $20.86 \pm 0.08$ & \\
\hline $1998 \mathrm{TF}_{35}$ & 2000 Nov. 29 & 19.25 & $23.06 \pm 0.08$ & $21.92 \pm 0.03$ & $21.24 \pm 0.06$ & $20.52 \pm 0.07$ & \\
\hline $1998 \mathrm{UR}_{43}$ & 2000 Nov. 29 & 31.65 & $\begin{array}{c}24.15 \pm 0.08 \\
-\end{array}$ & $\begin{array}{c}23.37 \pm 0.05 \\
-\end{array}$ & $\begin{array}{l}22.76 \pm 0.08 \\
23.00 \pm 0.08\end{array}$ & $\begin{array}{c}22.62 \pm 0.10 \\
-\end{array}$ & 2 \\
\hline $1998 \mathrm{WH}_{24}$ & 2000 Nov. 30 & 42.31 & $\begin{array}{c}22.23 \pm 0.07 \\
-\end{array}$ & $\begin{array}{c}21.22 \pm 0.03 \\
-\end{array}$ & $\begin{array}{l}20.63 \pm 0.06 \\
20.66 \pm 0.06\end{array}$ & $\begin{array}{c}20.05 \pm 0.07 \\
-\end{array}$ & \\
\hline $1998 \mathrm{WV}_{31}$ & 2000 Nov. 30 & 33.45 & $\begin{array}{c}24.17 \pm 0.08 \\
-\end{array}$ & $\begin{array}{c}23.34 \pm 0.05 \\
-\end{array}$ & $\begin{array}{l}22.81 \pm 0.07 \\
22.85 \pm 0.07\end{array}$ & $\begin{array}{c}22.47 \pm 0.10 \\
-\end{array}$ & \\
\hline $1998 \mathrm{WX}_{31}$ & 2000 Nov. 29 & 40.63 & - & $\begin{array}{c}22.86 \pm 0.06 \\
-\end{array}$ & $\begin{array}{l}22.24 \pm 0.07 \\
22.28 \pm 0.08\end{array}$ & $\begin{array}{c}21.62 \pm 0.10 \\
\simeq\end{array}$ & \\
\hline $1999 \mathrm{CC}_{158}$ & 2000 Nov. 29 & 42.43 & $\begin{array}{c}23.22 \pm 0.09 \\
-\end{array}$ & $\begin{array}{c}22.26 \pm 0.04 \\
-\end{array}$ & $\begin{array}{l}21.70 \pm 0.07 \\
21.68 \pm 0.06\end{array}$ & $\begin{array}{c}21.14 \pm 0.07 \\
-\end{array}$ & \\
\hline $1999 \mathrm{CD}_{158}$ & 2000 Nov. 30 & 48.32 & $23.06 \pm 0.07$ & $22.19 \pm 0.03$ & $21.71 \pm 0.06$ & $21.17 \pm 0.07$ & \\
\hline $1999 \mathrm{CF}_{119}$ & 2000 Nov. 30 & 39.41 & - & $\begin{array}{c}23.51 \pm 0.06 \\
-\end{array}$ & $\begin{array}{l}22.90 \pm 0.08 \\
23.01 \pm 0.07\end{array}$ & $\begin{array}{c}22.56 \pm 0.09 \\
-\end{array}$ & 2 \\
\hline $1999 \mathrm{DE}_{9}$ & 2000 Nov. 30 & 33.91 & $21.62 \pm 0.06$ & $20.76 \pm 0.03$ & $20.19 \pm 0.05$ & $19.63 \pm 0.06$ & \\
\hline $1999 \mathrm{OX}_{3}$ & 2000 Sep. 04 & 27.39 & $23.23 \pm 0.21$ & - & $21.28 \pm 0.06$ & $20.80 \pm 0.09$ & \\
\hline $1999 \mathrm{RZ}_{253}$ & 2000 Sep. 05 & 40.91 & - & $\begin{array}{c}22.13 \pm 0.05 \\
-\end{array}$ & $\begin{array}{l}21.48 \pm 0.05 \\
21.49 \pm 0.04\end{array}$ & $\begin{array}{c}20.84 \pm 0.05 \\
-\end{array}$ & \\
\hline $1999 \mathrm{TC}_{36}$ & $\begin{array}{l}2000 \text { Sep. } 05 \\
2000 \text { Nov. } 30\end{array}$ & 31.54 & $\begin{array}{c}21.46 \pm 0.03 \\
21.49 \pm 0.03 \\
21.58 \pm 0.05 \\
\simeq\end{array}$ & $\begin{array}{c}20.42 \pm 0.02 \\
20.43 \pm 0.02 \\
20.54 \pm 0.02 \\
-\end{array}$ & $\begin{array}{l}19.70 \pm 0.03 \\
19.76 \pm 0.03 \\
19.84 \pm 0.05 \\
19.84 \pm 0.05\end{array}$ & $\begin{array}{c}19.06 \pm 0.02 \\
19.12 \pm 0.02 \\
19.23 \pm 0.06 \\
\simeq\end{array}$ & 1 \\
\hline $1999 \mathrm{TD}_{10}$ & 2000 Sep. 05 & 12.38 & - & $19.97 \pm 0.01$ & $19.47 \pm 0.02$ & $19.00 \pm 0.02$ & 4,5 \\
\hline $1999 \mathrm{UG}_{5}$ & 2000 Nov. 29 & 8.00 & $\begin{array}{c}20.47 \pm 0.05 \\
-\end{array}$ & $\begin{array}{c}19.50 \pm 0.02 \\
-\end{array}$ & $\begin{array}{l}18.85 \pm 0.05 \\
18.83 \pm 0.05\end{array}$ & $\begin{array}{c}18.24 \pm 0.06 \\
-\end{array}$ & \\
\hline $2000 \mathrm{OK}_{67}$ & 2000 Nov. 29 & 40.80 & - & $\begin{array}{c}22.77 \pm 0.05 \\
-\end{array}$ & $\begin{array}{l}22.24 \pm 0.07 \\
22.26 \pm 0.07\end{array}$ & - & \\
\hline $2000 \mathrm{QC}_{243}$ & $\begin{array}{l}2000 \text { Nov. } 30 \\
2000 \text { Nov. } 29\end{array}$ & $\begin{array}{l}40.80 \\
19.40\end{array}$ & $\begin{array}{c}23.70 \pm 0.09 \\
21.99 \pm 0.06 \\
-\end{array}$ & $\begin{array}{c}22.97 \pm 0.05 \\
21.26 \pm 0.03 \\
-\end{array}$ & $\begin{array}{c}- \\
20.82 \pm 0.05 \\
20.81 \pm 0.05\end{array}$ & $\begin{array}{c}21.76 \pm 0.08 \\
20.42 \pm 0.06 \\
-\end{array}$ & \\
\hline
\end{tabular}

Notes: (1): contamination by a nearby star. The magnitudes presented in this table have been corrected for this effect, (2): possible variability, (3): aperture diameter of $3 F W H M,(4)$ : object weak in $B$ frame, (5): bad seeing for some of the frames. 

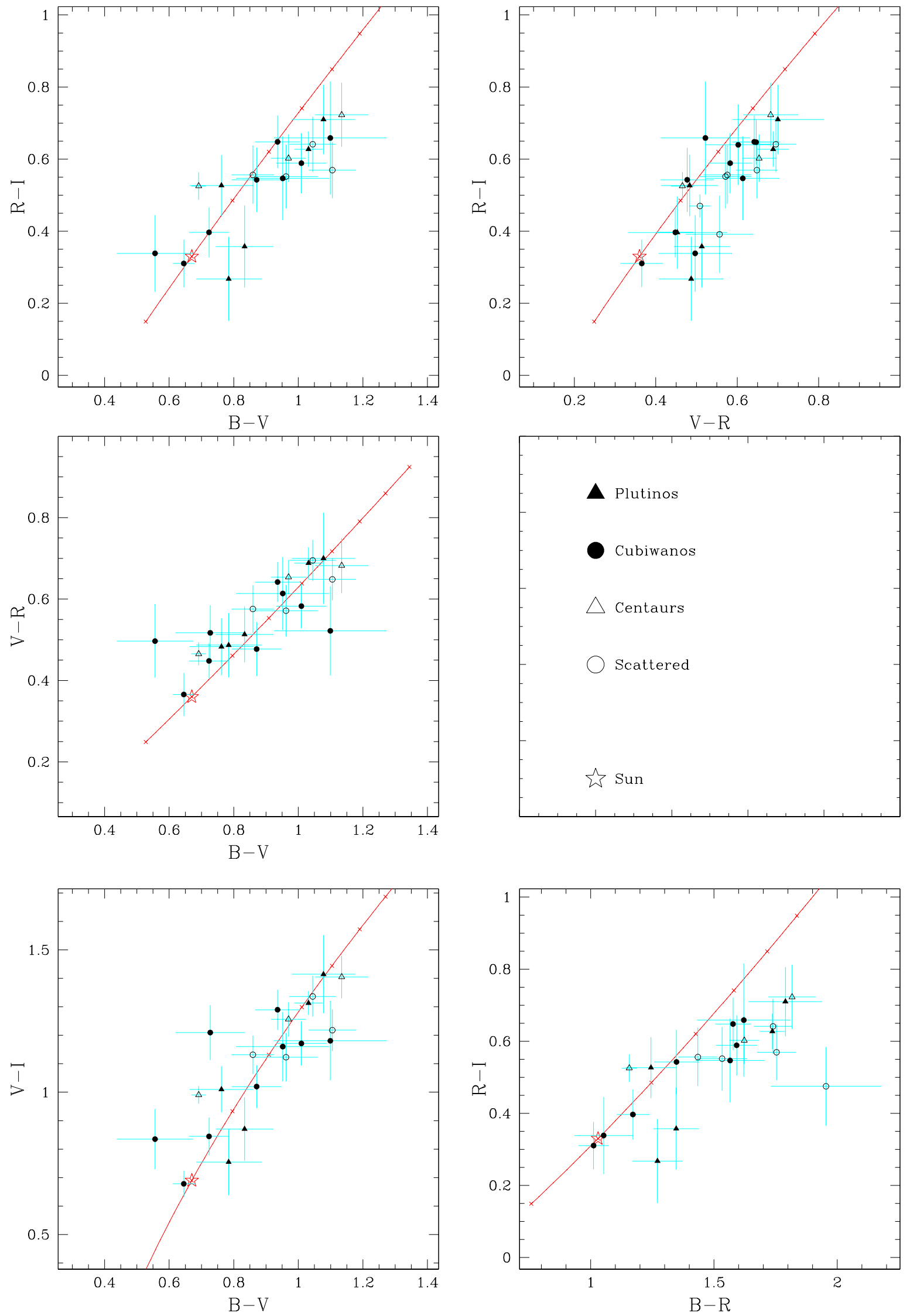

Fig. 1. Color-color diagrams for the 27 objects. The meaning of the different symbols is given in the middle right panel. The reddening line (which is the locus of objects displaying a linear reflectivity spectrum) has a range of gradients $\mathcal{S}$ from -10 to $70 \% / 100 \mathrm{~nm}$; a tick mark is placed at every 10 units. 
Table 3. Absolute magnitude and colors for all objects, obtained from the photometric measurements presented in Table 2 . For objects measured during both runs, we present one value per run, in order to highlight any instrinsic change.

\begin{tabular}{|c|c|c|c|c|c|c|c|c|}
\hline Object & (1) & $(2)$ & $M(1,1) \pm \sigma$ & $R_{N}[\mathrm{~km}]$ & $\mathcal{S} \pm \sigma$ & $B-V \pm \sigma$ & $V-R \pm \sigma$ & $R-I \pm \sigma$ \\
\hline $1993 \mathrm{SB}$ & Plut & $2 \mathrm{~b}$ & $8.07 \pm 0.08$ & $65.5 \pm 2.4$ & $13.31 \pm 3.78$ & $0.76 \pm 0.10$ & $0.48 \pm 0.07$ & $0.53 \pm 0.08$ \\
\hline \multirow[t]{2}{*}{1994 ТВ } & Plut & $1 \mathrm{~b}$ & $7.32 \pm 0.05$ & $92.5 \pm 2.5$ & $44.89 \pm 3.26$ & $1.10 \pm 0.07$ & $0.78 \pm 0.06$ & $0.72 \pm 0.08$ \\
\hline & & $2 \mathrm{~b}$ & $7.59 \pm 0.09$ & $81.8 \pm 3.6$ & $28.12 \pm 4.73$ & $1.04 \pm 0.12$ & $0.60 \pm 0.08$ & $0.70 \pm 0.12$ \\
\hline \multirow[t]{2}{*}{$1995 \mathrm{SM}_{55}$} & QB1 & $1 b$ & $4.39 \pm 0.02$ & $356.9 \pm 4.4$ & $0.27 \pm 1.92$ & $0.65 \pm 0.02$ & $0.38 \pm 0.03$ & $0.29 \pm 0.04$ \\
\hline & & $2 \mathrm{a}$ & $4.32 \pm 0.06$ & $368.0 \pm 10.2$ & $-1.14 \pm 3.58$ & $0.63 \pm 0.06$ & $0.33 \pm 0.06$ & $0.35 \pm 0.09$ \\
\hline $1995 \mathrm{TL}_{8}$ & Scat & $2 \mathrm{~b}$ & $4.58 \pm 0.06$ & $325.8 \pm 9.0$ & $33.94 \pm 3.05$ & $1.04 \pm 0.07$ & $0.69 \pm 0.05$ & $0.64 \pm 0.08$ \\
\hline $1996 \mathrm{RQ}_{20}$ & QB1 & $1 \mathrm{a}$ & $6.89 \pm 0.10$ & $112.4 \pm 5.7$ & $23.16 \pm 4.97$ & $0.95 \pm 0.14$ & $0.61 \pm 0.09$ & $0.55 \pm 0.12$ \\
\hline $1997 \mathrm{QH}_{4}$ & QB1 & $2 \mathrm{~b}$ & $7.19 \pm 0.13$ & $98.2 \pm 6.4$ & $20.81 \pm 6.34$ & $1.10 \pm 0.17$ & $0.52 \pm 0.11$ & $0.66 \pm 0.16$ \\
\hline $1997 \mathrm{QJ}_{4}$ & Plut & $1 b$ & $7.29 \pm 0.12$ & $93.6 \pm 5.3$ & $6.89 \pm 5.63$ & - & $0.45 \pm 0.12$ & $0.40 \pm 0.10$ \\
\hline $1998 \mathrm{BU}_{48}$ & Scat & $2 \mathrm{a}$ & $7.03 \pm 0.06$ & $105.5 \pm 3.0$ & $26.98 \pm 3.10$ & $1.10 \pm 0.07$ & $0.65 \pm 0.05$ & $0.57 \pm 0.08$ \\
\hline $1998 \mathrm{SG}_{35}$ & Cent & $1 b$ & $10.83 \pm 0.02$ & $18.4 \pm 0.2$ & $12.11 \pm 1.67$ & $0.69 \pm 0.02$ & $0.46 \pm 0.03$ & $0.52 \pm 0.04$ \\
\hline $1998 \mathrm{SM}_{165}$ & QB1 & $2 \mathrm{a}$ & $5.91 \pm 0.05$ & $176.6 \pm 4.7$ & $29.72 \pm 2.94$ & $0.94 \pm 0.07$ & $0.64 \pm 0.05$ & $0.65 \pm 0.07$ \\
\hline $1998 \mathrm{SN}_{165}$ & QB1 & $1 \mathrm{~b}$ & $5.44 \pm 0.09$ & $220.0 \pm 9.9$ & $7.83 \pm 4.78$ & $0.56 \pm 0.12$ & $0.50 \pm 0.09$ & $0.34 \pm 0.11$ \\
\hline $1998 \mathrm{TF}_{35}$ & Cent & $2 \mathrm{a}$ & $8.51 \pm 0.07$ & $53.4 \pm 1.8$ & $36.67 \pm 3.79$ & $1.13 \pm 0.08$ & $0.68 \pm 0.07$ & $0.72 \pm 0.09$ \\
\hline $1998 \mathrm{UR}_{43}$ & Plut & $2 \mathrm{a}$ & $7.94 \pm 0.08$ & $69.4 \pm 2.7$ & $5.73 \pm 4.66$ & $0.78 \pm 0.10$ & $0.49 \pm 0.08$ & $0.27 \pm 0.12$ \\
\hline $1998 \mathrm{WH}_{24}$ & QB1 & $2 \mathrm{~b}$ & $4.43 \pm 0.06$ & $350.0 \pm 10.5$ & $22.61 \pm 3.29$ & $1.01 \pm 0.08$ & $0.58 \pm 0.05$ & $0.59 \pm 0.08$ \\
\hline $1998 \mathrm{WV}_{31}$ & Plut & $2 \mathrm{~b}$ & $7.64 \pm 0.07$ & $79.7 \pm 2.7$ & $10.20 \pm 4.28$ & $0.83 \pm 0.09$ & $0.51 \pm 0.07$ & $0.36 \pm 0.11$ \\
\hline $1998 \mathrm{WX}_{31}$ & QB1 & $2 \mathrm{a}$ & $6.22 \pm 0.07$ & $153.1 \pm 5.6$ & $26.20 \pm 4.61$ & - & $0.60 \pm 0.08$ & $0.64 \pm 0.11$ \\
\hline $1999 \mathrm{CC}_{158}$ & Scat & $2 \mathrm{a}$ & $5.43 \pm 0.07$ & $220.7 \pm 8.0$ & $20.29 \pm 3.66$ & $0.96 \pm 0.10$ & $0.57 \pm 0.06$ & $0.55 \pm 0.09$ \\
\hline $1999 \mathrm{CD}_{158}$ & QB1 & $2 \mathrm{~b}$ & $4.90 \pm 0.07$ & $281.4 \pm 9.1$ & $13.43 \pm 3.73$ & $0.87 \pm 0.08$ & $0.48 \pm 0.06$ & $0.54 \pm 0.09$ \\
\hline $1999 \mathrm{CF}_{119}$ & Scat & $2 \mathrm{~b}$ & $7.03 \pm 0.08$ & $105.6 \pm 4.0$ & $13.45 \pm 4.60$ & - & $0.56 \pm 0.08$ & $0.39 \pm 0.11$ \\
\hline $1999 \mathrm{DE}_{9}$ & Scat & $2 \mathrm{~b}$ & $4.88 \pm 0.06$ & $284.4 \pm 8.0$ & $20.77 \pm 3.37$ & $0.86 \pm 0.07$ & $0.58 \pm 0.06$ & $0.56 \pm 0.08$ \\
\hline $1999 \mathrm{OX}_{3}$ & Scat & $1 \mathrm{a}$ & $6.97 \pm 0.22$ & $108.8 \pm 11.5$ & - & - & - & $0.47 \pm 0.11$ \\
\hline $1999 \mathrm{RZ}_{253}$ & QB1 & $1 b$ & $5.43 \pm 0.06$ & $284.4 \pm 7.8$ & $29.96 \pm 3.00$ & - & $0.65 \pm 0.06$ & $0.65 \pm 0.06$ \\
\hline \multirow[t]{2}{*}{$1999 \mathrm{TC}_{36}$} & Plut & $1 b$ & $4.83 \pm 0.03$ & $290.5 \pm 4.3$ & $31.69 \pm 1.70$ & $1.03 \pm 0.04$ & $0.68 \pm 0.03$ & $0.63 \pm 0.03$ \\
\hline & & $2 \mathrm{~b}$ & $4.88 \pm 0.05$ & $285.0 \pm 6.6$ & $33.51 \pm 2.64$ & $1.03 \pm 0.06$ & $0.70 \pm 0.04$ & $0.61 \pm 0.07$ \\
\hline $1999 \mathrm{TD}_{10}$ & Scat & $1 b$ & $8.71 \pm 0.02$ & $48.8 \pm 0.5$ & $12.83 \pm 1.50$ & - & $0.51 \pm 0.03$ & $0.47 \pm 0.03$ \\
\hline $1999 \mathrm{UG}_{5}$ & Cent & $2 \mathrm{a}$ & $10.09 \pm 0.04$ & $25.8 \pm 0.6$ & $28.82 \pm 2.58$ & $0.97 \pm 0.05$ & $0.65 \pm 0.04$ & $0.60 \pm 0.07$ \\
\hline \multirow[t]{3}{*}{$2000 \mathrm{OK}_{67}$} & QB1 & $2 \mathrm{a}$ & $6.14 \pm 0.06$ & $159.3 \pm 4.9$ & - & - & $0.52 \pm 0.07$ & - \\
\hline & & $2 \mathrm{~b}$ & - & $159.3 \pm 4.9$ & - & $0.73 \pm 0.11$ & - & - \\
\hline & & $(*)$ & $6.14 \pm 0.06$ & $159.3 \pm 4.9$ & $15.97 \pm 7.06$ & $0.73 \pm 0.11$ & $0.52 \pm 0.07$ & - \\
\hline $2000 \mathrm{QC}_{243}$ & QB1 & $2 \mathrm{a}$ & $7.95 \pm 0.05$ & $69.2 \pm 1.7$ & $6.96 \pm 2.72$ & $0.72 \pm 0.06$ & $0.45 \pm 0.04$ & $0.40 \pm 0.07$ \\
\hline
\end{tabular}

(1) class: QB1 = Cubewano, Plut = Plutino, Scat = Scaterred Disk Object, Cent = Centaur. (2) run (UT date): $1 \mathrm{a}=2000$ Sep. $04,1 b=2000$ Sep. $05,2 a=2000$ Nov. $29,2 b=2000$ Nov. $30\left(^{*}\right)=$ compilation of Run $2 \mathrm{a} \& 2 b$. M11 is the absolute $R$ magnitude. $R_{N}$ is an estimation of the radius of the object (in $\mathrm{km}$ ) given by Eq. (2). $\mathcal{S}$ is the spectral gradient in (\%/100 nm) as defined in the text.

where $M_{\odot}$ is the $R$ magnitude of the Sun, $M(1,1)$ is the absolute $R$ magnitude from Eq. (1) and $p$ is the albedo. The values obtained are also presented in Table 3.

It is interesting to note that this radius, evaluated assuming an albedo $p=0.04$, can be in error by a factor of 2 or more because of the uncertainty of this albedo. This is especially true for bluish objects, whose albedo could be fairly high (in the case of a fresh ice surface, i.e. 0.1 for Chiron, Hartmann et al. 1981), or very low (in the order of 0.02 in case of highly irradiated objects; Thompson et al. 1987). Therefore, this estimation of the radius has to be taken with precaution.
Table 4. Solar colors used in this paper, from Hardorp (1980); Campins et al. (1985) and Allen's Astrophysical Quantities Cox (2000).

\begin{tabular}{cc}
\hline Color & Value \\
\hline \hline$(B-V) \odot$ & 0.67 \\
$(V-R) \odot$ & 0.36 \\
$(V-I)_{\odot}$ & 0.69 \\
\hline
\end{tabular}

\subsection{Spectral gradient}

The color indexes can also be converted into relative spectral reflectivities $\mathcal{R}(\lambda)$, computed at the central 

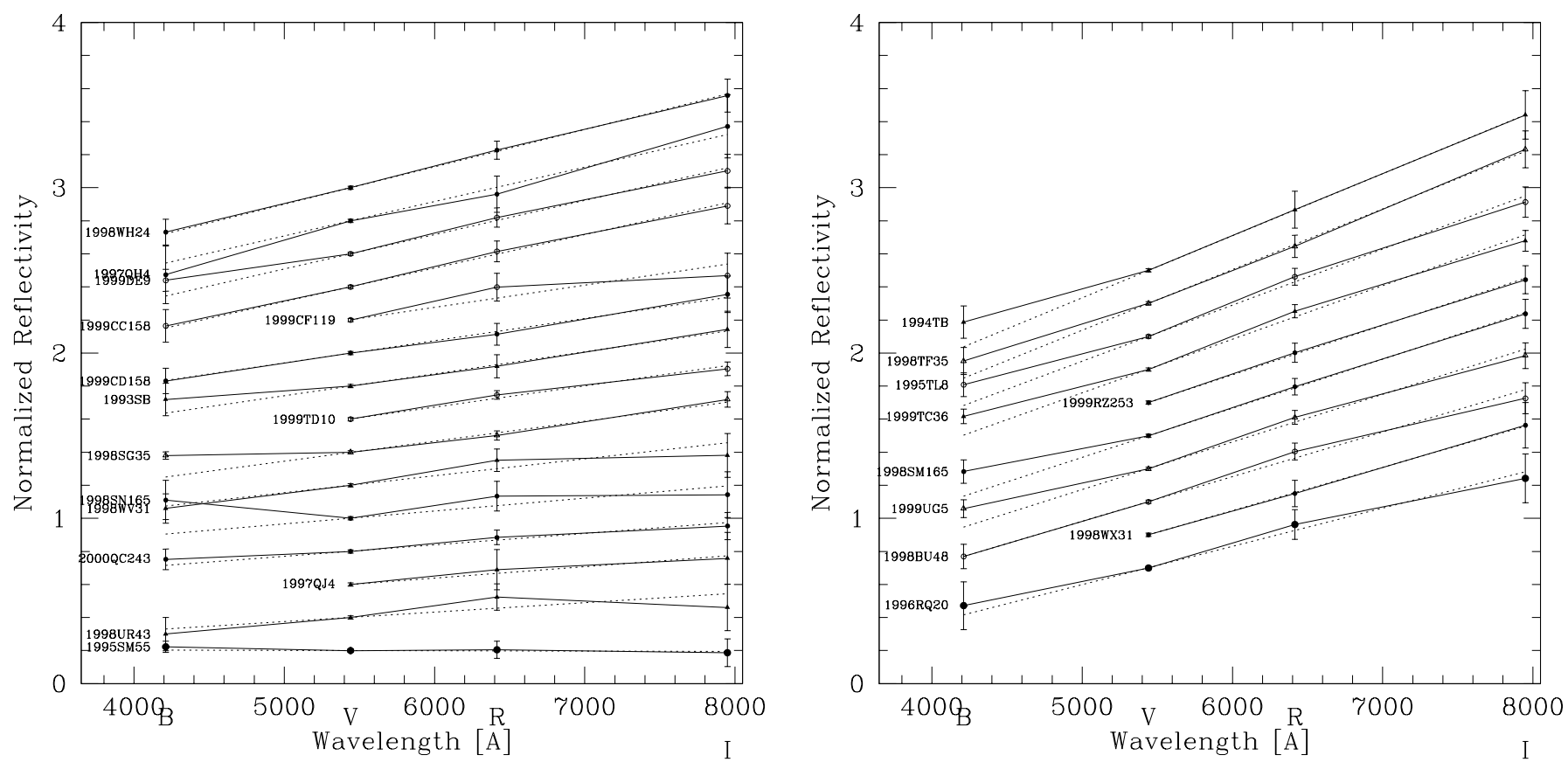

Fig. 2. Reflectivity spectra. The reflectivity is normalized to 1 for the $V$ filter; the "spectra" have been arbitrarily shifted for clarity. For each object, the dotted line is the linear regression over the $V, R, I$ range (excluding $B$ ), corresponding to the gradient $\mathcal{S}$ reported in this paper.

wavelength of the broadband filters used (Jewitt \& Meech 1986). We normalized these reflectivities at the central wavelength of the $V$ filter, leading to the following equation:

$\mathcal{R}(\lambda)=10^{-0.4[(m(\lambda)-m(V))-(m(\lambda)-m(V)) \odot],}$

where $m$ and $m_{\odot}$ are the magnitude of the object and of the Sun at the appropriate wavelength. In other words, the color indices are equivalent to very low resolution spectra. These spectra are presented in Fig. 2. To characterize a reflectivity spectrum, we can consider the reddening, $\mathcal{S}$ (also called the slope parameter or spectral index, cf. Hainaut \& Delsanti 2001), defined as percent of reddening per $100 \mathrm{~nm}$ as:

$\mathcal{S}\left(\lambda_{1}, \lambda_{2}\right)=100 \times \frac{\mathcal{R}\left(\lambda_{2}\right)-\mathcal{R}\left(\lambda_{1}\right)}{\left(\lambda_{2}-\lambda_{1}\right) / 1000}$.

As most objects have a linear reflectivity spectrum over the $V, R$, and $I$ bands, we can compute the mean reddening, $\mathcal{S}$, by linear regression. All the values are presented in Table 3 .

On each color-color plot we have represented a line which characterizes the locus of objects with a linear reflectivity spectrum, with reddening (i.e. spectral slope) ranging from $-10<\mathcal{S}<70 \% / 100 \mathrm{~nm}$, to guide the eye.

\section{Discussion}

This section compares our VLT data with those of merged sets compiled from the literature. It describes the possible group characteristics of the objects from color-color plots and outlines suggestions deduced from spectral gradient histograms of the objects.

\subsection{Color-color plots}

The color-color plots of the KBOs and Centaurs measured at the VLT (see Fig. 1) suggest the following:

The KBO color distributions appear to be rather uniform over the reddening range of $B-V \sim 0.55-1.1 \mathrm{mag}$, $V-R \sim 0.35-0.7 \mathrm{mag}$ and $R-I \sim 0.3-0.7 \mathrm{mag}$, respectively. A bimodal distribution (Tegler \& Romanishin $1998,2000)$ is not at all obvious in our data. A similar conclusion on the likely absence of a bimodal $B-V$ versus $V-R$ distribution of KBOs is already mentioned by previous photometric studies (Barucci et al. 1999; Davies et al. 2000; Boehnhardt et al. 2001). The question of the bimodality is discussed in detail in Hainaut \& Delsanti (2001) on the basis of the whole photometric database currently available (including those presented here) and with the help of different statistical tools. In summary, they demonstrate that, depending on the color index considered, there is either no bimodality at all, or that the limits of the bimodality are not statistically significant.

The reddening of the objects seems to be constant over the $B-V$ and $V-R$ wavelength range for a given object. Some objects show a deviation in the $B$ band; it must however be noted that these deviations are compatible with the larger error bars, caused by a lower $\mathrm{S} / \mathrm{N}$ ratio for the observations in this filter. The gradient systematically drops to smaller values for the $R-I$ color. This is indicated by $i$ ) the close alignment of the data points with the reddening line (see Fig. 1) in the $V-R$ versus $B-V$ plot, and ii) the drop of the data points below the line of constant reddening in 
the $R-I$ versus $B-V$ plot for the red objects. The latter effect suggests that the red objects have a flatter spectral gradient towards the far red and near-IR wavelength range while the neutral ones keep the reddening slope rather constant over the whole wavelength range measured. A similar result was already reported by Boehnhardt et al. (2001).

\subsection{Comparison of VLT data and merged dataset from literature}

This paper's dataset has been merged with a complete compilation of the TNO colors published in the literature (totalling $\sim 100$ objects); this compilation is presented and analyzed with a set of statistical tools in Hainaut \& Delsanti (2001). In this section, we shall highlight some of the comparisons between the VLT observations presented here and the whole, multi-telescope, multi-observer dataset (of which this dataset represent about $25 \%$ ).

Color-color diagrams and Dispersion of the data: the general aspect of the color-color diagrams is very similar. In particular, the reddest objects in the $B-V, R-I$ and $V-R, R-I$ also show a general trend to be lower than the reddening line (i.e. reflectivity spectrum less red at the longest wavelengths), as described in the previous paragraph. The general location and dispersion of the data is similar, indicating that both samples are compatible, i.e. no dramatic systematic effect is biasing some groups.

Outliers: both samples show some outliers, i.e. objects located very far from the general flock of objects group around the reddening line. In particular, $1998 \mathrm{SN}_{165}$, the most extreme outlier from our sample, was measured with similar colors by Gil-Hutton \& Licandro (2001).

Bimodality and statistical tests: the sample we present here, as well as the merged dataset, do not present obvious bimodality in the color distribution. Hainaut \& Delsanti (2001) have performed several statistical tests on the merged dataset, showing that there is no statistical support for a bimodal distribution (i.e. it is very unlikely that the observed distribution can be extracted from a bimodal distribution). The same tests, performed on this sample, would give the same negative result, but with a smaller significance.

\subsection{Spectral gradient statistics on merged dataset}

In Fig. 3, we show the distribution of the spectral gradients of KBOs and Centaurs. The histograms were compiled in the same way as described in Boehnhardt et al. (2001). The dataset used is composed of the measurements presented in this paper, combined with the input of Table 5 in Boehnhardt et al. (2001). The histogram bins
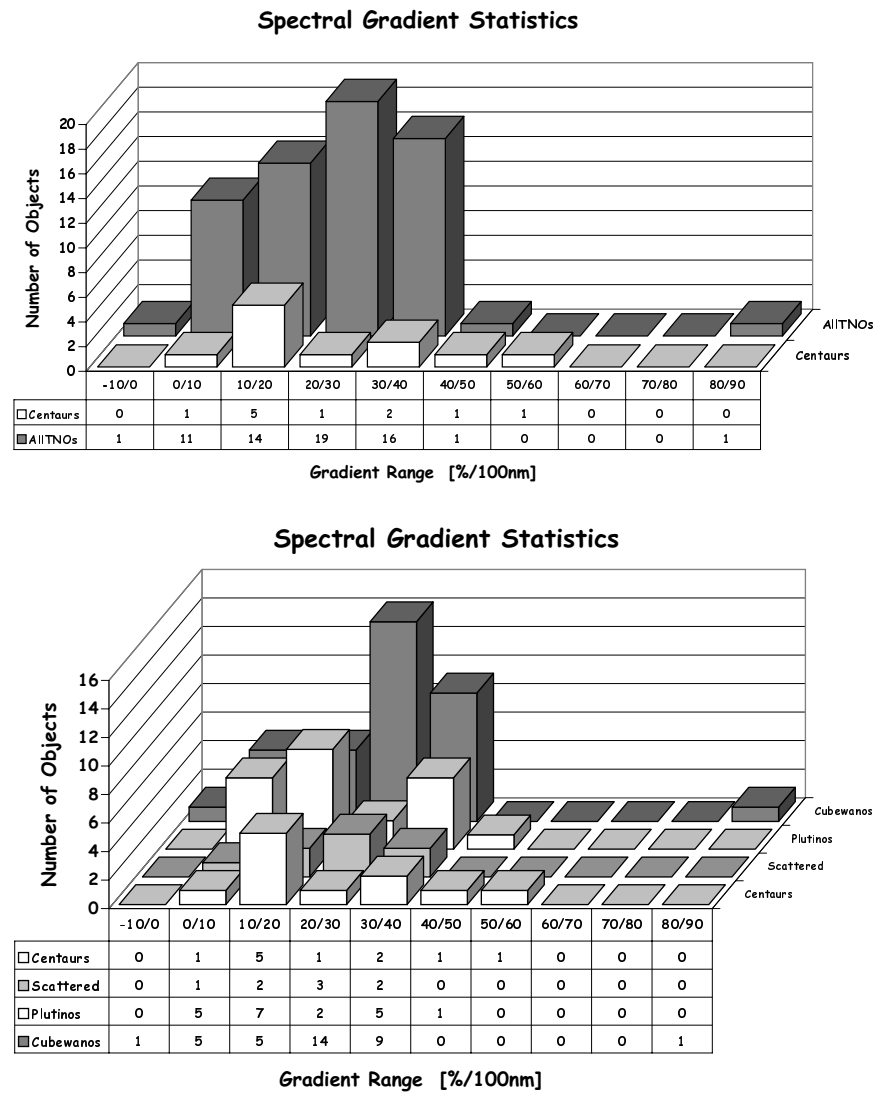

Fig. 3. Spectral gradient histograms. Upper panel: all TNOs and Centaurs, lower panel: Cubewanos, Plutinos, scattered disk objects and Centaurs. The histogram shows the number of objects per spectral gradient interval. The spectral gradient is given in \%/100 nm, the intervals are 10\%/100 nm wide which reflects the mean uncertainty of the spectral gradient data of the object sample. The tables in the bottom of the panels list the number of objects per spectral gradient interval for the various groups considered.

are $10 \% / 100 \mathrm{~nm}$ wide which is the mean uncertainty of the object spectral gradients.

The bulk of KBOs falls into the reddening range of $0-40 \% / 100 \mathrm{~nm}$ with the maximum objects towards the red end of the distribution. The Centaurs may have a distinctly different distribution of spectral gradients, since the distribution "peaks" between 10-20\%/100 nm. Considering the three dynamical groups among KBOs separately, it is obvious that the Cubewanos resemble best the global trend in the spectral gradient histograms of all KBOs, while the Plutinos seem to follow more the Centaurs' trend, i.e. with a peak towards smaller reddening gradients. A second "population" of Plutinos has very red spectral slopes, while the range of the Cubewano peak is poorly occupied among the Plutinos. It is too early to draw conclusions about the group properties of scattered disk objects since only 8 objects have been measured so far. For the same reasons of low statistical significance, the suggestions concerning the Centaur and Plutino population distribution peaks have to be viewed with caution. 


\subsection{Surface processing}

The evolution of the surface of the objects is probably the result of the balance of different processes. To date, the suspected processes are:

Aging: the surface of the objects, believed to be covered with organic-rich water ices, is irradiated by high-energy particles (cosmic rays ions, solar particles, etc.) and undergoes a progressive darkening due to the loss of hydrogen atoms, polymerization, and to aromatization of the mantle (Sagan et al. 1984; Johnson 2001; Moroz 2001). The time-scale "for a significant radiolytic evolution of the optical surface of a Kuiper Belt Object can vary from millions of years in the region of highest anomalous cosmic ray (ACR) intensity to a billion years elsewhere due to the modulated galactic cosmic ray flux", Johnson (2001). The possible effect of radiation on reflectance depends also on the dose. An initial bright ice mixture, which displays neutral colors, high albedo and a flat visible spectrum, will evolve into a material with a red spectrum at medium dose. Subsequently, this could lead to a neutral dark material with a lower albedo and a flat spectrum at a higher dose (Johnson 2001). An interesting question appears: can a surface element of an object remain undisturbed long enough to evolve from dark-red to neutral-black?

Collisions: impacts on the surface (Stern 1996) could modify the reflectance of the objects by excavating fresh material (i.e. non irradiated, with a neutral reflectance spectrum) from the interior. The resulting color is then the average of the surface, including the undisturbed aged ice and the freshly excavated ice (Luu \& Jewitt 1996).

Cometary activity: the active area can present fresh ice, possibly mixed with a rubble mantle. It is also possible that dust trapped by the gravity of the objects will recover part - or all - of the non active surface. The importance of cometary activity depends on the heliocentric distance of the object and on the thickness of the irradiated (and/or dust) mantle. However, observational evidence indicate that activity is routinely present at $15 \mathrm{AU}$ (Meech 1999). Activity could also be possible beyond $20 \mathrm{AU}$, as suggested by observations reported by Hainaut et al. (2000) and Fletcher et al. (2000) (although this result is only marginally significant), and by models presented by Prialnik (2001).

These three processes effect either the objects as a whole (aging) or only a fraction of the surface (nondisruptive collisions). However, at all times of its evolution, an object surface element will present a straight reflectivity spectrum at visible wavelengths (cf. laboratory spectra, Thompson et al. 1987). As a consequence, the average reflectivity spectrum of the object is also straight, explaining why all the objects are within their error bars on the reddening line (which represents the locus

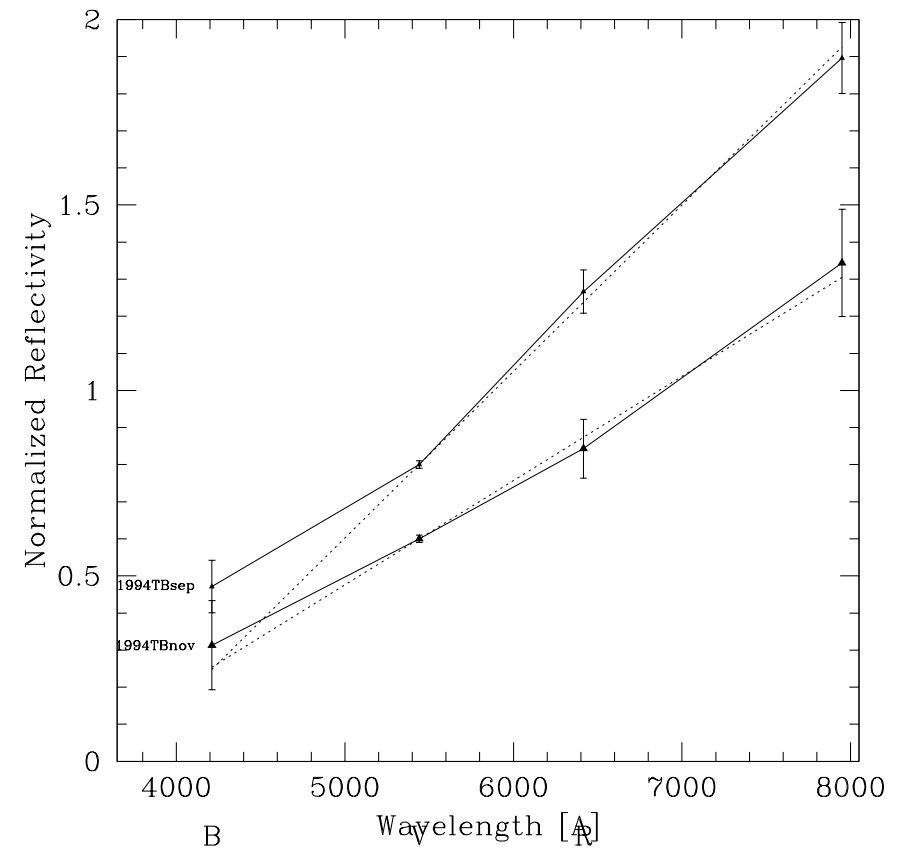

Fig. 4. Reflectivity spectrum of 1994 TB during the two runs. The reflectivity is normalized to 1 for the $V$ filter; the spectra have been arbitrarily shifted for clarity. The dotted line is the linear regression over the $V, R, I$ range, corresponding to the gradient $\mathcal{S}$. The change of mean slope (from 44.9 to $28.1 \% / 100 \mathrm{~nm}$ between the 2 runs) is the result of significant changes in the colors, indicating that this variation is real.

in a color-color plot of objects with a linear reflectivity spectrum).

\subsection{Overview of the objects}

In this section, we compare our color values with those reported in the literature. We also mention the other information available for these objects. This study is presented in Table 5; some additional comments can be found below for some of the objects.

1994 TB: there is a significant decrease of brightness by $0.25-0.30 \mathrm{mag}$ between September and November (in $R$ and $I$ band), which is about 2 times the error bars. This could be due to rotational effects. To date, no light-curve has been published. We present the reflectivity spectra at the two different epochs in Fig. 4. It is also interesting to note that the colors and spectral gradient of the object changed significantly between the 2 epochs, suggesting a variegated surface. This object deserves a detailed multi-color light-curve.

$1995 \mathbf{S M}_{\mathbf{5 5}}$ : the absolute magnitude is marginally brightening by 0.07 magnitude (including a solar phase effect correction of $-\beta \alpha$ of $\left.0.008 \mathrm{mag} /{ }^{\circ}\right)$. The gradients are compatible between the two periods (cf. Table 3 ).

It is interesting to note that Hillier et al. (2001) observed $1995 \mathrm{SM}_{55}$ several times in $J$ band over the Oct. 1999-Jan. 2001 period. The object's J light-curve 
Table 5. Overview of the objects and comparison with data available in the literature.

\begin{tabular}{|c|c|c|c|c|c|c|}
\hline \multirow[t]{2}{*}{ Objects } & \multirow[t]{2}{*}{$(1)$} & \multicolumn{4}{|r|}{ Our data } & \multirow{2}{*}{$\begin{array}{l}\text { Other published } \\
\text { information }\end{array}$} \\
\hline & & $\begin{array}{c}\mathrm{cf} \\
\text { text }\end{array}$ & $\begin{array}{l}\text { Gradient } \\
\text { Color }\end{array}$ & $(2)$ & Comparison with other data ${ }^{(3)}$ & \\
\hline $1993 \mathrm{SB}$ & Plut & & Neutral & & $\begin{array}{l}V-R \text { compatible with Gil01 } \\
B-V, V-R \text { compatible with Teg00 } \\
V-R, R-I \text { comp. with Lag00 }(1 \sigma) \\
\text { R mag compatible with Wil95 }\end{array}$ & Dav00: $V-J$ color \\
\hline $1994 \mathrm{~TB}$ & Plut & $\bar{\checkmark}$ & Red & $\mathrm{v}$ & $\begin{array}{l}B V R I \text { comp. with Luu96 \& Teg97 } \\
B-V, V-R: \text { good agreem. with Bar99 } \\
V-R, R-I \text { comp. with Lag00 }(1 \sigma) \\
V-R \text { compatible with Teg98 }\end{array}$ & Dav00: V-J color \\
\hline $1995 \mathrm{SM}_{55}$ & QB1 & $\checkmark$ & $\begin{array}{l}\text { Neutral } \\
\text { Bluish }\end{array}$ & $\mathrm{v}$ & $\begin{array}{l}V-R \text { compatible with Gil01 } \\
B V R I \text { colors compatible with Boe01 } \\
\text { (Run } 2 \text { at } 1.5 \sigma \text { level) }\end{array}$ & $\begin{array}{l}\text { Boe01: visible spectrum } \\
\text { Hil01: } J \text { light-curve }\end{array}$ \\
\hline $1995 \mathrm{TL}_{8}$ & Scat & & Red & & First published & \\
\hline $1996 \mathrm{RQ}_{20}$ & QB1 & $\checkmark$ & Red & $\mathrm{v}$ & $\begin{array}{l}B V R I \text { colors compatible with Boe } 01 \\
V-R 0.17 \mathrm{mag}>\operatorname{Teg} 98(1.5 \sigma)\end{array}$ & \\
\hline $1997 \mathrm{QH}_{4}$ & QB1 & & Neutral & & $\begin{array}{l}\text { Teg00: } B-V \text { equal, } V-R \text { compatible }(1 \sigma) \\
R-I \text { first published }\end{array}$ & \\
\hline $1997 \mathrm{QJ}_{4}$ & Plut & & $\begin{array}{l}\text { Neutral } \\
\text { Bluish }\end{array}$ & & $\begin{array}{l}V-R \text { in agreement with Gil01 } \\
B-V, R-I \text { first published }\end{array}$ & Dav00: $V-J$ color \\
\hline $1998 \mathrm{BU}_{48}$ & Scat & $\checkmark$ & Red & & First published & She01: light-curve \\
\hline $1998 \mathrm{SG}_{35}$ & Cent & & Neutral & & $\begin{array}{l}\text { Dor01: } V-R, R-I \text { compatible; Our } \\
B-V<0.2 \mathrm{mag} \text { (margin. signif.) }\end{array}$ & \\
\hline $1998 \mathrm{SM}_{165}$ & QB1 & & Red & & $B-V, V-R$ margin. comp. with Teg00 & \\
\hline $1998 \mathrm{SN}_{165}$ & QB1 & & $\begin{array}{l}\text { Neutral } \\
\text { Bluish }\end{array}$ & & $\begin{array}{l}V-R \text { compatible with Gil01 } \\
\text { Dor01: } V-R, R-I \text { margin. compatible; } \\
\text { Our } B-V<0.25 \text { mag (margin. signif.) }\end{array}$ & \\
\hline $1998 \mathrm{TF}_{35}$ & Cent & & Very red & & $B-V, V-R$ compatible with Boe01 & \\
\hline $1998 \mathrm{UR}_{43}$ & Plut & $\bar{\checkmark}$ & $\begin{array}{c}\text { Neutral } \\
\text { Bluish }\end{array}$ & $\mathrm{v}$ & $V-R<0.13$ mag than Gil01 & \\
\hline $1998 \mathrm{WH}_{24}$ & QB1 & & Red & & $\begin{array}{l}B-V, V-R \text { compatible with Teg00 } \\
V-R \text { marginally comp. with Boe } 01\end{array}$ & $\begin{array}{l}\text { Bro01: "Pholus-like" (4) } \\
\text { near-IR spectrum }\end{array}$ \\
\hline $1998 \mathrm{WV}_{31}$ & Plut & & Neutral & & First published & \\
\hline $1998 \mathrm{WX}_{31}$ & QB1 & & Red & & First published & \\
\hline $1999 \mathrm{CC}_{158}$ & Scat & & Neutral & & First published & \\
\hline $1999 \mathrm{CD}_{158}$ & QB1 & & Neutral & & First published & \\
\hline $1999 \mathrm{CF}_{119}$ & Scat & $\checkmark$ & Neutral & $\mathrm{v}$ & First published & \\
\hline $1999 \mathrm{DE}_{9}$ & Scat & $\checkmark$ & Neutral & & First published & $\begin{array}{l}\text { She01: light-curve } \\
\text { Bro01: "Pholus-like" }{ }^{(4)} \text { spec. }\end{array}$ \\
\hline $1999 \mathrm{OX}_{3}$ & Scat & & Neutral & & $R-I$ first published & Dor01: $B-V, V-R$ \\
\hline $1999 \mathrm{RZ}_{253}$ & QB1 & & Red & & $V R I$ first published & $\begin{array}{l}\text { Dor01: } B-V \\
\text { Bro00: near-IR spectrum }\end{array}$ \\
\hline $1999 \mathrm{TC}_{36}$ & Plut & & Red & & $\begin{array}{l}B V R I \text { colors compatible with Boe } 01 \\
\text { Dor01: } B-V, V-R \text { compatible; } \\
\text { Our } R-I<0.1 \text { mag }(1 \sigma)\end{array}$ & Bro00: near-IR spectrum \\
\hline $1999 \mathrm{TD}_{10}$ & Scat & & Neutral & & $V-R$ compatible with Con00 & Bro01: "Pholus-like" (4) spec. \\
\hline $1999 \mathrm{UG}_{5}$ & Cent & $\checkmark$ & Red & & $\begin{array}{l}\text { In agreement with Gut01 } \\
\text { and marginally with Pei01 }\end{array}$ & Gut01: $R$ light-curve \\
\hline $2000 \mathrm{OK}_{67}$ & QB1 & $\checkmark$ & Neutral & $\mathrm{v}$ & First published & \\
\hline $2000 \mathrm{QC}_{243}$ & QB1 & & $\begin{array}{c}\text { Neutral } \\
\text { Bluish }\end{array}$ & & First published & \\
\hline
\end{tabular}

(1) Class: QB1 = Cubewano, Plut $=$ Plutino, Scat $=$ Scaterred Disk Object, Cent $=$ Centaur.

(2) Object displaying a variability during the VLT observations.

(3) Abbreviation code for the references: Bar99 = Barucci et al. (1999), Boe01 = Boehnhardt et al. (2001), Bro00 = Brown (2000), Bro01 = Brown (2001), Con00 = Consolmagno et al. (2000), Dav00 = Davies et al. (2000), Dor01 = Doressoundiram et al. (2001), Gil01 = Gil-Hutton \& Licandro (2001), Gut01 = Gutiérrez et al. (2001), Hil01 = Hillier et al. (2001), Luu96 = Luu \& Jewitt (1996), Lag00 = Lagerkvist et al. (2000), Pei01 = Peixinho et al. (2001), She01 = Sheppard \& Jewitt (2001), Teg97 = Tegler \& Romanishin (1997), Teg98 = Tegler \& Romanishin (1998), Teg00 = Tegler \& Romanishin (2000), Wil95 = Williams et al. (1995).

(4) "Pholus-like" spectrum : characterized by 2 features of water ice and a feature of methanol, Brown (2001). 
is compatible with a constant, except for rapid variations (about 0.4 mag in a few hours) on 8 October 1999 and during January 2000, and for a brightening of almost 1 mag on 22 October, 1999. While the brightening could possibly be explained by mediocre observing conditions, the authors affirm that the rapid changes seen over the January 2000 period are real and that they must correspond to a physical process occurring on the object.

Our observations, corrected for $V-J$, are compatible with the "quiescent" phase of the object. Considering the neutral-bluish colors of the object, the short time-scale of the variations observed and their seemingly random behavior, $1995 \mathrm{SM}_{55}$ could possibly host some cometary activity, and deserves a detailed study.

$1996 \mathbf{R Q}_{\mathbf{2 0}}$ : displays a marginally significant variability in $R$ over half an hour.

$1998 \mathrm{BU}_{48}$ : no variability within the same night. Sheppard \& Jewitt (2001) report a light-curve with an amplitude of $0.4 \mathrm{mag}$ and a period larger than 4 hours.

$1998 \mathbf{U R}_{43}$ : significant variation in $R$ band of $\sim 0.3$ mag over $\sim 1$ hour suggesting a light-curve effect. Our $V-R$ value is 0.13 lower than that of Gil-Hutton $\&$ Licandro (2001). As our measurements is right on the reddening line, it is possible that their color was affected by the rotation.

$1999 \mathbf{C F}_{119}$ : shows a variability of $0.1 \mathrm{mag}$ in the $R$ band over half an hour, suggesting a rotational effect.

1999 DE: : Sheppard \& Jewitt (2001) report a lightcurve constant within 0.05 mag over few hours. Jewitt et al. (2000) reported a spectrum showing water ice features; however, this result has not been published yet.

$1999 \mathrm{UG}_{5}$ : a light-curve in $R$ band has been published by Gutiérrez et al. (2001). The authors reported noticeable changes in brightness ( $0.2 \mathrm{mag}$ ) over 5 hours, which may be interpreted as the rotational light-curve (they cannot discriminate whether the variations are caused by the shape or by an albedo feature). Other causes are still possible, although cometary activity does not seem very likely.

$2000 \mathbf{O K}_{\mathbf{6 7}}$ : the repeated observations in $V$ band indicate a significant variation of 0.2 mag between the two runs.

\subsection{Discussion}

As explained in Sect. 5.4, the locus of objects with a linear spectrum (but with different spectral slopes) is the "reddening line" shown in the color-color plots (Fig. 1) for a slope $\mathcal{S}$ ranging from -10 to $70 \% / 100 \mathrm{~nm}$. The objects presented in this paper are located along this line (within their error bars, see Fig. 1), indicating that they display linear reflectivity spectra over the visible (VRI) wavelengths.

We could expect that an object undergoing an aging process caused by irradiation (i.e. reddening of its surface, cf. Sect. 6.4) may move along this line toward redder colors. At even higher irradiation dose, objects display a neutral but darker reflectivity spectrum (Thompson et al. 1987; Johnson 2001), which means that they should move back down toward more neutral colors along this reddening line on the color-color plots. It is however unclear if a TNO can remain undisturbed long enough to evolve into a dark neutral colored object.

More generally, the possible path of a generic Kuiper Belt object in a color-color plot during its life has still to be determined, possibly by the modeling of the contribution of the different evolution processes involved (i.e. not only by the aging process). We can also consider that bluish objects are likely to be covered by fresh ice (in the case they are not covered by high-irradiated neutral-dark material Thompson et al. 1987), and are therefore more likely to host some cometary activity. As neutral objects could therefore have very young surfaces, with possible cometary activity, or very old surfaces with a very low albedo, we recommend observers to devote telescope time to these objects, especially with instruments that could directly measure their albedo (e.g. the thermal emission) such as SIRTF, or detect a cometary activity (e.g. very high spatial resolution with a large collecting area.)

\section{Summary}

We have presented the photometry of an important set of objects (24 TNOs and 3 Centaurs, which represent $\sim 1 / 3$ of the published objects to date) over the visible range (BVRI) from two run of observations (Sep. and Nov. 2000) with an $8 \mathrm{~m}$ telescope (ESO/VLT). The objects display a broad and continuous range of colors from neutral-bluish to very red, with no evidence of a bimodal color distribution. The distribution of the objects in the color-color plots shows that, within the error bars, the KBOs display linear reflectivity spectra over the visible $(V R I)$ wavelengths.

Our measurements are compatible with those in the literature. For some of the objects, the availability of several epochs allowed us to detect some possible intrinsic changes such as for $1994 \mathrm{~TB}, 1995 \mathrm{SM}_{55}, 1998 \mathrm{UR}_{43}, 1999 \mathrm{CF}_{119}$ and $2000 \mathrm{OK}_{67}$. It appears that some objects deserve a complete study, such as $1995 \mathrm{SM}_{55}$ (to look for any possible cometary activity) or 1994 TB (for its spectral slope variability).

No further statistical test was performed on this dataset; a very detailed study of a larger sample including all published colors to date (including the data from this paper) is available in Hainaut \& Delsanti (2001).

\section{References}

Barucci, M. A., Doressoundiram, A., Tholen, D., Fulchignoni, M., \& Lazzarin, M. 1999, Icarus, 142, 476

Barucci, M. A., Fulchignoni, M., Birlan, M., et al. 2001, A\&A, 371,1150

Barucci, M. A., Romon, J., Doressoundiram, A., \& Tholen, D. J. 2000, AJ, 120, 496

Boehnhardt, H., Tozzi, G. P., Birkle, K., et al. 2001, A\&A, 378,653

Brown, M. 2001, Meudon KBO workshop, June 2001 
Brown, M. E. 2000, in AAS/Division of Planetary Sciences Meeting, vol. 32, 2008

Brown, R. H., Cruikshank, D. P., \& Pendleton, Y. 1999, ApJ, 519, L101

Brown, R. H., Cruikshank, D. P., Pendleton, Y. J., \& Veeder, G. J. 1997, Science, 276, 937

Bus, S. J., A'Hearn, M. F., Schleicher, D. G., \& Bowell, E. 1991, Science, 251, 774

Campins, H., Rieke, G. H., \& Lebofsky, M. J. 1985, ApJ, 90, 896

Consolmagno, G. J., Tegler, S. C., Rettig, T., \& Romanishin, W. 2000, Bull. Am. Astron. Soc., 32

Cox, A. N. 2000, Allen's Astrophysical Quantities (New York: Springer)

Cruikshank, D. P., Roush, T. L., Bartholomew, M. J., et al. 1998, Icarus, 135, 389

Davies, J. K., Green, S., McBride, N., et al. 2000, Icarus, 146, 253

Davies, J. K., Sykes, M. V., \& Cruikshank, D. P. 1993, Icarus, 102,166

Delahodde, C. E., Meech, K. J., Hainaut, O. R., \& Dotto, E. 2001, A\&A, 376, 672

Doressoundiram, A., Barucci, M. A., Romon, J., \& Veillet, C. 2001, Icarus, in press

ESO 2000, MIDAS, Munich Image and Data Analysis System, URL http://www.eso.org/projects/esomidas/midasoverview.html

ESO 2001a, The FORS Intruments, URL http://www . eso.org/instruments/fors1/index.html

ESO 2001b, The Very Large Telescope Project, URL http://www.eso.org/projects/vlt/

Fletcher, E., Fitzsimmons, A., Williams, I. P., Thomas, N., \& Ip, W.-H. 2000, in Minor Bodies in the Outer Solar System, ed. A. Fitzsimmons, D. Jewitt, \& R. M. West (Springer)

Gil-Hutton, R., \& Licandro, J. 2001, Icarus, submitted

Gutiérrez, P. J., Ortiz, J. L., Alexandrino, E., Roos-Serote, M., \& Doressoundiram, A. 2001, A\&A, 371, L1

Hainaut, O. R. 2001, Midas TMAG Photometry Package, URL http://sc6.sc.eso.org/ ohainaut

Hainaut, O. R., Delahodde, C. E., Boehnhardt, H., et al. 2000, A\&A, 356, 1076

Hainaut, O. R., \& Delsanti, A. C. 2001, A\&A, submitted

Hainaut, O. R., Meech, K. J., Boehnhardt, H., \& West, R. M. 1998, A\&A, 333, 746

Hardorp, J. 1980, A\&A, 88, 334

Hartmann, W. K., Cruikshank, D. P., Degewij, J., \& Capps, R. W. 1981, Icarus, 47, 333

Hillier, J., Green, S., Davies, J., McBride, N., \& Tholen, D. 2001, Meudon KBO workshop, June 2001

Jewitt, D., Luu, J., \& Trujillo, C. 2000, in AAS/Division of Planetary Sciences Meeting, vol. 32, 2007
Jewitt, D., \& Meech, K. J. 1986, ApJ, 310, 937

Johnson, R. 2001, Meudon KBO workshop, June 2001

Keller, H. U. 1990, in Physics and Chemistry of Comets, ed. Huebner (Springer Verlag), 13

Lagerkvist, C., Dahlgren, M., Ekholm, A., et al. 2000, in Minor Bodies in the Outer Solar System, ed. A. Fitzsimmons, D. Jewitt, \& R. M. West (Springer)

Landolt, A. U. 1992, AJ, 104, 340

Levison, H. 2001, in Highlights in Astronomy, IAU Joint Discussion 4, ed. H. Rickman, in press

Luu, J., \& Jewitt, D. 1996, AJ, 112, 2310

Luu, J. X., Jewitt, D. C., \& Trujillo, C. 2000, ApJ, 531, L151

Meech, K. J. 1999, in Evolution and Source Regions of Asteroids and Comets, IAU Colloq., 173, 195

Meech, K. J., \& Jewitt, D. C. 1987, A\&A, 187, 585

Minor Planet Center 2001a, List Of Centaurs and ScatteredDisk Objects, URL http://cfa-www.harvard.edu/iau/lists/Centaurs.html

Minor Planet Center 2001b, Observable Distant Minor Planets, URL http://cfa-www.harvard.edu/iau/ Ephemerides/Distant/index.html

Minor Planet Center 2001c, Unusual Minor Planets, URL http://cfa-www.harvard.edu/iau/lists/Unusual.html

Moroz, L. 2001, Meudon KBO workshop, June 2001

Noll, K. S., Luu, J., \& Gilmore, D. 2000, AJ, 119, 970

Peixinho, N., Lacerda, P., Ortiz, J. L., et al. 2001, A\&A, 371, 753

Prialnik, D. 2001, Meudon KBO workshop, June 2001

Russell, H. N. 1916, ApJ, 43, 173

Sagan, C., Khare, B. N., \& Lewis, J. S. 1984, Saturn, ed. Matthews \& Gehrels (Tucson: University of Arizona Press), 788

Sheppard, S., \& Jewitt, D. 2001, Meudon KBO workshop, June 2001

Stern, S. A. 1996, AJ, 112, 1203

Tegler, S. C., \& Romanishin, W. 1997, Icarus, 126, 212

Tegler 1998, Nature, 392, 49

Tegler 2000, Nature, 407, 979

Thomas, N., Eggers, S., Ip, W., et al. 2000, in Minor Bodies in the Outer Solar System, Proc. of the ESO Workshop held at Garching, Germany, 2-5 November 1998, ed. A. Fitzsimmons, D. Jewitt, \& R. M. West (Springer), 25

Thompson, W. R., Murray, B. G. J. P. T., Khare, B. N., \& Sagan, C. 1987, J. Geophys. Res., 92, 14933

Trujillo, C., Bouchez, A., \& Brown, M. 2001, Meudon KBO workshop, June 2001

Williams, I. P., O'Ceallaigh, D. P., Fitzsimmons, A., \& Marsden, B. G. 1995, Icarus, 116, 180 